\title{
The correlation between endometrial thickness and pregnancy outcomes in fresh ART cycles with different age groups: a retrospective study
}

\author{
Maryam Eftekhar ${ }^{1,2}$, Sara Zare Mehrjardi ${ }^{3}$, Behnaz Molaei ${ }^{4}$, Fatemeh Taheri ${ }^{1}$ and Esmat Mangoli ${ }^{{ }^{*}}$
}

\begin{abstract}
Background: In assisted reproductive technology (ART) cycles in addition to embryo quality, the receptivity of the endometrium plays the main role in clinical outcomes. Endometrial receptivity is necessary to implantation of an embryo, and ultrasound has been established as an appreciated, simple, and non-invasive technique in the evaluation of the endometrial preparation before embryo transfer in fresh in vitro fertilization (IVF) cycles. Debate on the predictive value measuring endometrial thickness before administering human chorionic gonadotropin (HCG) for ovulation triggering in ART is ongoing. In order to explore the impact of endometrial thickness on triggering day on ongoing pregnancy rate (OPR) in ART cycles, we retrospectively analyzed data from 1000 patients undergoing IVF/ICSI cycles.

Results: The data showed pregnancy rate was increased in the endometrial thickness of $8 \mathrm{~mm}$ to $11 \mathrm{~mm}$ then decreased, and in endometrial thickness (Ent) $>14 \mathrm{~mm}$, pregnancy rate was zero. There were significant differences in endometrial thickness and pregnancy outcomes between different age groups. The pregnancy rate was higher (32\%) in 23-30 years old women, and the range of Ent in this group was 6-12 mm. Also, the data showed a positive correlation between Ent with $\mathrm{AMH}$ and estradiol levels and the number of COC and MII oocytes and a negative correlation between female age with Ent.

Conclusion: The result showed that Ent on hCG administration day is associated with pregnancy outcomes in fresh IVF/ICSI cycles with different age groups, although some of the clinical parameters may have an effect on Ent. Large studies are needed to make a definitive conclusion.
\end{abstract}

Keywords: Endometrial thickness, ART, Female age, Pregnancy

\section{Background}

Assisted reproductive technology has been usually used in infertility treatment over the past three decades. In addition to embryo quality, the receptivity of the endometrium plays a main role in clinical outcome [1]. Endometrial receptivity is necessary to implantation of an embryo, and ultrasound has been established as an appreciated, simple, and non-invasive technique in evaluation of endometria preparation before embryo transfer

\footnotetext{
* Correspondence: es.mangoli@gmail.com

${ }^{1}$ Research and clinical center for infertility, Yazd Reproductive Sciences Institute, Shahid Sadoughi University of Medical Science, Yazd, Iran Full list of author information is available at the end of the article
}

in fresh IVF cycles. Several sonographic parameters have been assessed that include endometrial thickness (Ent), endometrial pattern (EnP), endometrial volume, and sub-endometrial blood flow [2]. Trans-vaginal ultrasonography (TVU) is often used to measure Ent on the day of HCG administration as the best echogenic space between the junction of the endometrium and myometrium in the mid-sagittal plane. It has been widely suggested that a thin endometrium is related to lower IVF-ICSI pregnancy rates, but an agreement is still lacking on what the exact definition of thin endometrium [3]. Debate on the predictive value measuring Ent before administering HCG for ovulation triggering in assisted 
reproduction techniques is ongoing. Some of the investigators have shown a linear correlation between pregnancy rates and Ent, though others have posited that pregnancy rates may even deteriorate above a thickness of $14 \mathrm{~mm}$ and then miscarriage rates may increase [4]. In order to explore the impact of endometrial thickness on triggering day on ongoing pregnancy rate (OPR) in IVF-ET cycles, we retrospectively analyzed data from 1000 patients with different age groups undergoing IVF/ ICSI cycles.

\section{Methods}

The study was a retrospective study and was done by reviewing the clinical data of fresh IVF/ICSI cycles at the Yazd Research and Clinical Center for infertility from May 2016 until May 2018. The study included women aged 18-45 years without endometriosis. Also, patients with severe male factor infertility, patients with a known endometrial polyp or uterine anomaly, oocyte donation cycles, and preimplantation genetic diagnosis (PGD) cycles were excluded.

\section{Ovarian stimulation}

Ovarian stimulation, sperm preparation, IVF embryo culture, and fresh ET protocols in our center have been described elsewhere $[5,6]$. Briefly, according to the maternal age, cause of infertility, ovarian response, and coexisting medical conditions, agonist and antagonist ovarian stimulation protocols were done [6]. Recombinant human chorionic gonadotropin (hCG) $(250 \mathrm{mg}$; Ovidrel, Serono, Geneva) or urinary HCG $(10,000 \mathrm{IU}$, Pregnyl, Organon, Netherland) was administered to trigger ovulation when two leading follicles reached a mean diameter of $17 \mathrm{~mm}$. Trans-vaginal ultrasound-guided oocyte retrieval was conducted 36 after hCG administration. Fertilization of the oocytes took place either by IVF or ICSI, according to the sperm quality. The luteal phase was supported by vaginal progesterone (Cyclogest, Actavis, UK), $400 \mathrm{mg}$, and twice a day. One or two embryos were transferred 2 days later. Fertilization rate was defined as the percentage of fertilized oocytes (2PN) to all mature oocytes (MII). Chemical pregnancies were confirmed 2 weeks after embryo transfer, by positive serum $\beta$ HCG measurement. Clinical pregnancy was defined by the presence of gestational sac in the uterus, 4 weeks after embryo transfer. The implantation rate estimated by the number of gestational sacs that visualized on TVU divided by the number of embryos transferred [6].

\section{Endometrial assessment}

Ent was measured by TVU on the day of triggering. In the longitudinal plane of the uterus, the maximum distance between the two interfaces of endometrium- myometrium junction was defined as the thickness of endometrium [7].

\section{Statistical analysis}

Results are showed as the mean \pm standard deviation (SD) or percentage (\%). The means of samples with normal distribution and of sufficient size were compared by Student's $t$ test and one-way ANOVA. Differences between abnormal scatterings and the medians were compared by Mann-Whitney $U$ and Kruskal-Wallis test. Chisquare $\left(\chi^{2}\right)$ test was employed to assess the differences between categorical variables. A $p$ value $<0.05$ was considered significant. Statistical analysis was performed using the Statistical Package for the Social Sciences 20 (SPSS Inc., Chicago, IL, USA).

\section{Results}

One thousand fresh IVF/ICSI cycles were recruited in this study, among which 277 cycles achieved chemical pregnancy $(27.7 \%)$, and from them, 245 cycles achieved clinical pregnancy $(88.4 \%)$.

To explore the relationship between endometrial thickness and chemical and clinical pregnancy rates in all the 1000 cycles, patients were divided into 9 distinct groups according to the endometrial thickness on the day of embryo transfer: $\leq 7 \mathrm{~mm}, 7-8 \mathrm{~mm}, 8-9 \mathrm{~mm}$, 9$10 \mathrm{~mm}, 10-11 \mathrm{~mm}, 11-12 \mathrm{~mm}, 12-13 \mathrm{~mm}, 13-14 \mathrm{~mm}$, and $\geq 14 \mathrm{~mm}$. Chemical and clinical pregnancy rates were calculated for each endometrial thickness interval.

There was a significant correlation between Ent and pregnancy outcomes $(p<0.0001)$. Also, the data show that by increasing the endometrium thickness to $11 \mathrm{~mm}$, pregnancy increases and then decreases (Table 1, Fig. 1).

There were significant differences in clinical parameters like female age, AMH, estradiol level on the day of final triggering, and the numbers of retrieved oocytes

Table 1 Comparison of pregnancy outcomes in different endometrial thickness

\begin{tabular}{lll}
\hline Endometrial thickness $(\mathrm{mm})$ & $\begin{array}{l}\text { Chemical pregnancy } \\
\text { rate }(\%)\end{array}$ & $\begin{array}{l}\text { Clinical pregnancy } \\
\text { rate }(\%)\end{array}$ \\
\hline$<7$ & $5(7.4)$ & $5(7.4)$ \\
$7-8$ & $26(13.2)$ & $24(12.2)$ \\
$8-9$ & $86(29.2)$ & $75(25.4)$ \\
$9-10$ & $90(31.2)$ & $77(26.6)$ \\
$10-11$ & $33(42.9)$ & $29(37.7)$ \\
$11-12$ & $14(25)$ & $11(19.6)$ \\
$12-13$ & $3(33.3)$ & $3(33.3)$ \\
$13-14$ & $1(25)$ & $1(25)$ \\
$>14$ & $0(0)$ & $0(0)$ \\
$p$ value & $<0.0001^{*}$ & $<0.0001^{*}$ \\
\hline
\end{tabular}

${ }^{*} p$ value $<0.05$ was considered to be significant 


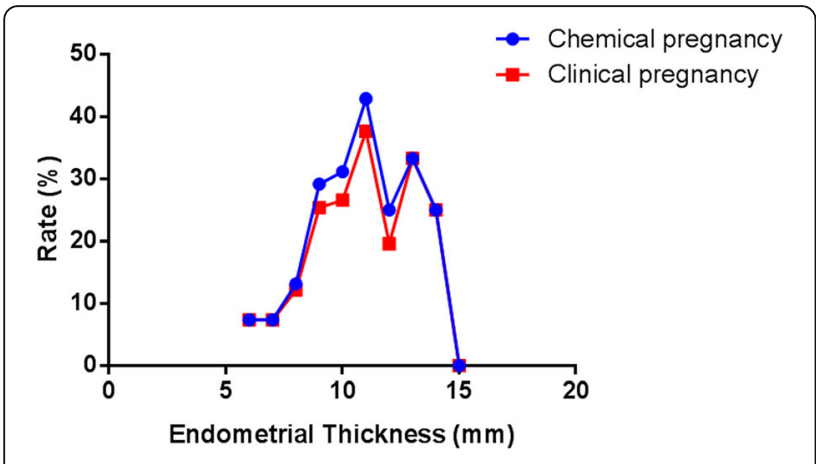

Fig. 1 The relationship between endometrial thickness and pregnancy outcomes

and oocyte maturity in pregnant patients compared to non-pregnant (Table 2).

Also, for determining the effect of female age on the pregnancy rate and the relationship with endometrial thickness, we divided patients to 4 different age groups: $18-23,23-30,30-38$, and $>38$. The data showed the rate of pregnancy was significantly different between different age groups $(p=0.001)$, and in group 2 were higher than others. Also, when we compared endometrial thickness between different age groups, we saw that the range of Ent in group 2 was 6-12 $\mathrm{mm}$ that confirms our results in Table 1 (Tables 3 and 4).

The data showed a positive correlation between Ent with $\mathrm{AMH}$ and estradiol levels and the number of $\mathrm{COC}$ and MII oocytes. On the other hand, there is a negative correlation between female age and Ent (Table 5).

\section{Discussion}

We had studied fresh IVF/ICSI cycle of 1000 patients and determined the effect of Ent on the day of final triggering on pregnancy outcome. Our results showed that there is a significant correlation between endometrial thickness and pregnancy rate.

While several studies have confirmed the association of thin endometrium with low clinical pregnancy rate, the Ent cutoff point for IVF-ET pregnancy outcome has not been identified. Studies showed that the clinical pregnancy rate and ongoing pregnancy rate are significantly reduced when Ent is less than $7 \mathrm{~mm}$ on the hCG day $[7,8]$ that was in line with our study. It was reported that spontaneous abortion rate is significantly increased when Ent $>14 \mathrm{~mm}$ [9], whereas another study proposes there is no adverse effect on pregnancy outcome when Ent $>14 \mathrm{~mm}$ [7]. In our study, we observed that pregnancy rate was increased from Ent $=8 \mathrm{~mm}$ to Ent $=11 \mathrm{~mm}$, then decreased, and in Ent $>14 \mathrm{~mm}$, the pregnancy rate was zero.

In order to get a more accurate relationship between endometrial thickness and IVF outcome, $\mathrm{Bu}$ et al. demonstrated that for the patient undergoing IVF with the different ovarian response, a thin endometrial thickness on HCG administration day is associated with lower pregnancy rate [10]. A thick endometrium is more controversial. Several previous investigators have suggested that a thick endometrium $(>16$, or $\geq 17 \mathrm{~mm}$ ) is associated with an improved conception rate, whereas others have reported a detrimental effect of a thick endometrium $(>14 \mathrm{~mm})$ on conception rate $[11,12]$. Yuan et al., in their study, found a consistently positive correlation between Ent and conception rate, with patients having an Ent greater than $15 \mathrm{~mm}$ achieving the highest conception rate of $53.3 \%$ [3]. It is important to note, however, that a thickened endometrium is associated with a number of intrauterine pathologies such as polyps or fibroids, both of which have been shown to adversely affect implantation and reduce pregnancy rates [13]. Therefore, in women with an abnormally thickened endometrium, it seems prudent to carry out further investigations, such as a hysteroscopy to rule out and treat any intra-cavity lesions.

Vânia Costa Ribera and coworker in a study evaluated whether Ent affects the outcome of a fresh embryo transfer, in a retrospective, single-center cohort of 3350 IVF cycles. In the multivariate regression analysis, Ent was nonlinearly associated with live birth, live birth rates being the lowest with an Ent less than $7.0 \mathrm{~mm}(21.6 \% ; p<0.001)$ and then between 7.0 and $9.0 \mathrm{~mm}(30.2 \% ; p=0.008)$. An Ent less than $7.0 \mathrm{~mm}$ was also associated with a decrease in neonatal birth weight $z$-scores. They concluded that the use of Ent is a potential prognostic tool for live birth rates and neonatal birth weight [4].

Table 2 The relationship between clinical parameters and pregnancy outcomes

\begin{tabular}{llll}
\hline Variables & Chemical pregnancy & Clinical pregnancy & $p$ value \\
\hline Female age & $31.50 \pm 5.8$ & $29.49 \pm 5.0$ & $0.001^{*}$ \\
AMH & $6.03 \pm 5.80$ & $6.00 \pm 5.70$ & $<0.0001^{*}$ \\
Estradiol & $2300 \pm 2590$ & $2300 \pm 2501$ & $<0.0001^{*}$ \\
The number of COC & $11.00 \pm 12.00$ & $11.00 \pm 12.00$ & $0.001^{*}$ \\
The number of MII & $9.50 \pm 11.00$ & $8.00 \pm 11.00$ & $<0.0001^{*}$ \\
\hline
\end{tabular}

The data was nonparametric and presented with median \pm interquartile range

${ }^{*} p$ value $<0.05$ was considered to be significant 
Table 3 The rate of pregnancy between different age groups

\begin{tabular}{llllll}
\hline Variables & Group 1, N=99 & Group 2, N=444 & Group 3, N=362 & Group 4, N=95 & $p$ value \\
\hline Chemical pregnancy (\%) & $30(30.3)$ & $144(32.5)$ & $94(26)$ & $9(9.5)$ & $0.001^{*}$ \\
Clinical pregnancy (\%) & $27(28.2)$ & $134(30.1)$ & $76(21.5)$ & $8(8.5)$ & $0.001^{*}$ \\
\hline
\end{tabular}

Group 1: 18-23 years old; group 2: 23-30 years old; group 3: 30-38 years old; and group 4: > 38 years old. The data were presented with percent ${ }^{*} p$ value $<0.05$ was considered to be significant

Mohammady and coworker in their study evaluated the effect of combined endometrial thickness and pattern on the success of intracytoplasmic sperm injection (ICSI) cycles and concluded endometrial thickness of $10-12.9 \mathrm{~mm}$ with trilaminar pattern is associated with higher CPR with ICSI cycles [14].

Julian et al. in their study evaluated the effect of endometrial pattern at triggering day on implantation [15]. The endometrial pattern (Enp) was recorded as being in one of the following three categories, as described by Grunfeld et al. [16]: (1) late proliferative (hyperechoic endometrium constituting $<50 \%$ of the Ent, with a hyperechoic basalis and hypoechoic functional), (2) early secretory (hyperechoic basalis and functional extending to $>50 \%$ of the Ent, but not covering the entire endometrial cavity), and (3) mid-late secretory (homogeneous hyperechoic functionalize extending from the basalis to the lumen). Within the study population, Ent was not significantly associated with clinical outcomes of euploid ETs. A type 3 Enp at trigger day suggests a prematurely closed window of implantation [15].

Zhao and coworker showed a significant difference in endometrial thickness on the day of HCG administration and the change in endometrial thickness from the third day of gonadotropin stimulation to the day of HCG administration between pregnant women and nonpregnant women. This finding indicates that adequate endometrial development is favorable for improved pregnancy rate. They propose that the endometrium of non-pregnant women may be associated with pathological abnormalities, such as squamous cell metaplasia or lacking a normal proliferative response to the rising estradiol level. In a binary logistic regression model, the endometrial thickness on day 3 of gonadotropin stimulation, endometrial thickness on the day of HCG administration, and the change in endometrial thickness from the third day of gonadotropin stimulation to the day of HCG administration were independent predictive factors for pregnancy [1] Although many studies have evaluated the relationship between endometrial thickness and IVF outcome, the results are still controversial. Some authors reported no relationship between endometrial thickness and pregnancy rate in patients undergoing IVF $[17,18]$.

Because of patient basic parameters, such as the number of oocytes retrieved, quality and the number of embryos transferred were not fine assessed in most studies, and the results from them were questionable. In our study, we showed that there is a significant difference between clinical parameters like $\mathrm{AMH}$, estradiol level on the day of final triggering, and the numbers of retrieved oocytes and mature oocytes in the pregnant and nonpregnant patients. Also, there was a positive correlation between Ent with these clinical parameters. Ent is a crucial factor for pregnancy, but there is a possibility that these parameters may be more important than Ent and may have an effect on it. In fact, ovarian reserve and internal estradiol production cause endometrial growth. $\mathrm{AMH}$ is considered a sign of the ovarian reserve; for instance, it is generally expressed by those follicles that are newly progressive from the primordial follicle pool. Hence, serum AMH levels are demonstrative of the number and feature of the ovarian follicular pool [19]. Also, AMH is related to the number of mature oocytes and fertilization rates. On the other hand, there was a negative correlation between Ent and female age. We found that female age is an important factor in fertility,

Table 4 Comparison of endometrial thickness between different age groups of pregnant patients

\begin{tabular}{|c|c|c|c|c|c|}
\hline Variables & Group 1 & Group 2 & Group 3 & Group 4 & $p$ value \\
\hline Endometrial thickness (mm) & $9.21 \pm 1.17,9(6-14)$ & $9.19 \pm 1.31,9(6-12)$ & $8.86 \pm 1.38,9(5-15)$ & $8.44 \pm 1.93,8(5.5-15)$ & $\begin{array}{l}<0.0001^{a, f} \\
0.999^{b} \\
0.148^{c} \\
0.001^{d, e} \\
0.041^{g}\end{array}$ \\
\hline
\end{tabular}

Group1: 18-23 years old; group 2: 23-30 years old; group 3: 30-38 years old; and group 4: > 38 years old. The data were presented with mean \pm SD, median (min-max)

${ }^{\text {a }}$ The difference between groups

${ }^{\mathrm{b}}$ The difference between groups 1 and 2

'The difference between groups 1 and 3

${ }^{\mathrm{d}}$ The difference between groups 1 and 4

'The difference between groups 2 and 3

${ }^{f}$ The difference between groups 2 and 4

${ }^{9}$ The difference between groups 3 and 4 
Table $\mathbf{5}$ Correlation of endometrial thickness with different clinical parameters

\begin{tabular}{llllll}
\hline Variables & Female age & AMH & Estradiol & COC & MII \\
\hline Endometrial thickness & $r=-0.194, p<0.0001$ & $r=0.168, p<0.0001$ & $r=0.231, p<0.0001$ & $r=0.238, p<0.0001$ & $r=0.239, p<0.0001$ \\
\hline
\end{tabular}

Correlation was significant at 0.01 levels

and its increase, although negligibly, leads to a reduction in the clinical pregnancy rate. Keane et al. in line with our study showed that both AFC and AMH are strongly dependent on patient age and can estimate IVF outcomes, including pregnancy and live birth rates [20]. Reichman and colleagues showed that AMH is a properly strong metric for the prediction of ART cancelation and how many oocytes may be retrieved after stimulation, but conversely, they showed it was a moderately poor test for the prediction of pregnancy after any given treatment cycle. Patients with extremely low levels of $\mathrm{AMH}$ still can achieve reasonable treatment outcomes and should not be precluded from attempting IVF solely on the basis of an AMH value [21]. Studies showed that an increasing number of total oocytes, zygotes, and the quality of embryos are associated with live birth following donor oocyte IVF cycles [10, 22]. Barton et al. found that age and E2 on the day of hCG administration were predictive of mature oocytes and embryos retrieved, but FSH was not [23].

\section{Conclusion}

Our study demonstrated that Ent in hCG administration day correlated with pregnancy outcomes in fresh IVF/ ICSI cycles. The highest pregnancy rate was accompanied by $8-11 \mathrm{~mm}$ endometrial thicknesses and decreased to zero when Ent was $>14 \mathrm{~mm}$. Also, there was a positive correlation between Ent and clinical parameters and can affect pregnancy outcomes. On the other hand, we found the female age had a negative correlation with Ent and pregnancy outcomes.

\footnotetext{
Abbreviations

AMH: Anti-Mullerian hormone; ART: Assisted reproductive technology; CPR: Clinical pregnancy rate; EnP: Endometrial pattern; Ent: Endometrial thickness; ET: Embryo transfer; HCG: Human chorionic gonadotropin; ICSI: Intracytoplasmic sperm injection; IVF: In vitro fertilization; OPR: Ongoing pregnancy rate; TVU: Trans-vaginal ultrasonography
}

\section{Acknowledgements}

Not applicable

\section{Authors' contributions}

$\mathrm{ME}, \mathrm{SZ}$, and BM designed the study and contributed to the acquisition of data. FT and EM analyzed and interpreted the data. ME and EM were the main persons in writing the manuscript. All authors read and approved the final manuscript.

\section{Funding}

Not applicable.

\section{Availability of data and materials}

The datasets used and/or analyzed during the current study are available from the corresponding author on reasonable request.
Ethics approval and consent to participate

The study was approved by the Scientific and Ethics Committee of Islamic Azad University, Yazd Branch, Yazd, Iran (IR. SSU.MEDICINE.REC.1394.338).

\section{Consent for publication}

Not applicable

\section{Competing interests}

The authors declare that they have no competing interests.

\section{Author details}

${ }^{1}$ Research and clinical center for infertility, Yazd Reproductive Sciences Institute, Shahid Sadoughi University of Medical Science, Yazd, Iran. ${ }^{2}$ Abortion Research Center, Yazd Reproductive Sciences Institute, Shahid Sadoughi University of Medical Science, Yazd, Iran. ${ }^{3}$ Faculty of Medical Sciences, Islamic Azad University, Yazd Branch, Yazd, Iran. ${ }^{4}$ Department of obstetrics and Gynecology, Zanjan University of Medical Science, Zanjan, Iran.

Received: 4 August 2019 Accepted: 2 December 2019

Published online: 18 December 2019

\section{References}

1. Zhao J, Zhang Q, Wang Y, Li Y (2014) Endometrial pattern, thickness and growth in predicting pregnancy outcome following 3319 IVF cycle. Reprod BioMed Online 29(3):291-298

2. Kader MA, Abdelmeged A, Mahran A, Samra MFA, Bahaa H (2016) The usefulness of endometrial thickness, morphology and vasculature by $2 \mathrm{D}$ Doppler ultrasound in prediction of pregnancy in IVF/ICSI cycles. Egypt J Radiol Nucl Med 47(1):341-346

3. Yuan X, Saravelos SH, Wang Q, Xu Y, Li T-C, Zhou C (2016) Endometrial thickness as a predictor of pregnancy outcomes in 10787 fresh IVF-ICSI cycles. Reprod BioMed Online 33(2):197-205

4. Ribeiro VC, Santos-Ribeiro S, De Munck N, Drakopoulos P, Polyzos NP, Schutyser $V$ et al (2018) Should we continue to measure endometrial thickness in modern-day medicine? The effect on live birth rates and birth weight. Reprod BioMed Online 36(4):416-426

5. Eftekhar M, Firouzabadi RD, Karimi H, Rahmani E (2012) Outcome of cryopreserved-thawed embryo transfer in the GnRH agonist versus antagonist protocol. Iran J Reprod Med 10(4):297

6. Eftekhar M, Mohammadian F, Yousefnejad F, Khani P (2013) Microdose $\mathrm{GnRH}$ agonist flare-up versus ultrashort $\mathrm{GnRH}$ agonist combined with fixed $\mathrm{GnRH}$ antagonist in poor responders of assisted reproductive techniques cycles. Int J Fertil Steril 6(4):266

7. Fang R, Cai L, Xiong F, Chen J, Yang W, Zhao X (2016) The effect of endometrial thickness on the day of hCG administration on pregnancy outcome in the first fresh IVF/ICSI cycle. Gynecol Endocrinol 32(6):473-476

8. Eftekhar M, Tabibnejad N, Tabatabaie AA (2017) The thin endometrium in assisted reproductive technology: an ongoing challenge. Middle East Fertil Soc J 23(1):1-7

9. Weissman A, Gotlieb L, Casper RF (1999) The detrimental effect of increased endometrial thickness on implantation and pregnancy rates and outcome in an in vitro fertilization program. Fertil Steril 71(1):147-149

10. Bu Z, Sun $Y$ (2015) The impact of endometrial thickness on the day of human chorionic gonadotrophin ( $\mathrm{hCG}$ ) administration on ongoing pregnancy rate in patients with different ovarian response. PLoS One 10(12): e0145703

11. Al-Ghamdi A, Coskun S, Al-Hassan S, Al-Rejjal R, Awartani K (2008) The correlation between endometrial thickness and outcome of in vitro fertilization and embryo transfer (IVF-ET) outcome. Reprod Biol Endocrinol 6(1):37

12. Richter KS, Bugge KR, Bromer JG, Levy MJ (2007) Relationship between endometrial thickness and embryo implantation, based on 1,294 cycles of in vitro fertilization with transfer of two blastocyst-stage embryos. Fertil Steril 87(1):53-59 
13. Pritts EA, Parker WH, Olive DL (2009) Fibroids and infertility: an updated systematic review of the evidence. Fertil Steril 91(4):1215-1223

14. Al Mohammady M, Fattah GA, Mahmoud M (2013) The impact of combined endometrial thickness and pattern on the success of intracytoplasmic sperm injection (ICSI) cycles. Middle East Fertil Soc J 18(3):165-170

15. Gingold JA, Lee JA, Rodriguez-Purata J, Whitehouse MC, Sandler B, Grunfeld L et al (2015) Endometrial pattern, but not endometrial thickness, affects implantation rates in euploid embryo transfers. Fertil Steril 104(3):620-8. e5

16. Grunfeld L, Walker B, Bergh PA, Sandler B, Hofmann G, Navot D (1991) Highresolution endovaginal ultrasonography of the endometrium: a noninvasive test for endometrial adequacy. Obstet Gynecol 78(2):200-204

17. Bassil S (2001) Changes in endometrial thickness, width, length and pattern in predicting pregnancy outcome during ovarian stimulation in in vitro fertilization. Ultrasound Obstet Gynecol 18(3):258-263

18. De Geyter C, Schmitter M, De Geyter M, Nieschlag E, Holzgreve W, Schneider HP (2000) Prospective evaluation of the ultrasound appearance of the endometrium in a cohort of 1,186 infertile women. Fertil Steril 73(1): 106-113

19. Peluso C, Fonseca FL, Rodart IF, Cavalcanti V, Gastaldo G, Christofolini DM et al (2014) AMH: an ovarian reserve biomarker in assisted reproduction. Clin Chim Acta 437:175-182. https://doi.org/10.1016/j.cca.2014.07.029

20. Keane K, Cruzat VF, Wagle S, Chaudhary N, Newsholme P, Yovich J (2017) Specific ranges of anti-Mullerian hormone and antral follicle count correlate to provide a prognostic indicator for IVF outcome. Reprod Biol 17(1):51-59. https://doi.org/10.1016/j.repbio.2016.12.002

21. Reichman DE, Goldschlag D, Rosenwaks Z (2014) Value of antimullerian hormone as a prognostic indicator of in vitro fertilization outcome. Fertil Steril 101 (4):1012-8 e1. https://doi.org/10.1016/j.fertnstert.2013.12.039

22. Hariton E, Kim K, Mumford SL, Palmor M, Bortoletto P, Cardozo ER et al (2017) Total number of oocytes and zygotes are predictive of live birth pregnancy in fresh donor oocyte in vitro fertilization cycles. Fertil Steril 108(2):262-268. https://doi.org/10.1016/j.fertnstert.2017.05.021

23. Barton SE, Missmer SA, Ashby RK, Ginsburg ES (2010) Multivariate analysis of the association between oocyte donor characteristics, including basal follicle stimulating hormone (FSH) and age, and IVF cycle outcomes. Fertil Steril 94(4):1292-1295. https://doi.org/10.1016/j.fertnstert.2009.07.1672

\section{Publisher's Note}

Springer Nature remains neutral with regard to jurisdictional claims in published maps and institutional affiliations.

\section{Submit your manuscript to a SpringerOpen ${ }^{\circ}$ journal and benefit from:}

- Convenient online submission

- Rigorous peer review

- Open access: articles freely available online

- High visibility within the field

- Retaining the copyright to your article

Submit your next manuscript at $\boldsymbol{\nabla}$ springeropen.com 